\title{
Evaluación del grado de adecuación del proceso de forja en caliente para la fabricación de piezas estampadas: criterios de viabilidad y optimización ${ }^{(\cdot)}$
}

\author{
José Manuel Arenas Reina*, Miguel Angel Sebastián Pérez**, Pedro J. Nuñez López*** \\ y Cristina González Gaya**
}

\begin{abstract}
Resumen El diseño de piezas industriales supone un claro ejemplo de esfuerzo integrador que, con un enfoque interdisciplinar, requiere un análisis detallado de los diferentes conocimientos científicos y tecnológicos implicados en su concepción y desarrollo. Dentro de este marco de actuación, el presente trabajo aborda la problemática del proyecto de piezas estampadas en caliente, proponiendo la utilización de criterios de viabilidad y optimización que, fundamentados en consideraciones técnico-económicas, permiten valorar el grado de adecuación de las diferentes alternativas al proceso de forja, facilitando con ello la selección de la más adecuada. Fijadas estas bases y con objeto de acortar tiempos de respuesta y análisis en una aplicación correspondiente a piezas axisimétricas, se ha introducido un sistema informatizado que, tomando como dato de partida el plano de mecanizado de la pieza, simula geométricamente el proceso de estampación, permitiendo calcular los coeficientes de adecuación en aquellas tipologías que son posibles según los criterios de viabilidad. Este sistema también proporciona una representación gráfica de pieza mecanizada, pieza estampada y matriz de acabado.
\end{abstract}

Palabras clave Forja en caliente. Proyecto de piezas estampadas. Diseño para la fabricabilidad. Viabilidad. Optimización.

\section{Evaluation of the hot forging adjustment to the manufacture of forged parts: criteria of viability and optimization}

The design of industrial parts is a clear example of integrative effort that, with a focus of
several disciplines, requires a detailed analysis of the different scientific and technological
knowledge implied in its conception and development. Inside this performance context,
the present work approaches the problem of the project of hot forging parts, proposing the
use of criteria of viability and optimization that, based in technical and economical
considerations, allow to valuate the adjusting degree of various alternative to the forge
process, facilitating with it the selection of the most appropriate. Fixed these bases and with
object of shortening times of answer and analysis in application corresponding to parts
with revolution symmetry, a computerized system has been introduced that, taking as
started data the design of the part, geometrically simulates the forge process, allowing to
calculate the adaptation coefficients in those morphologing that are possible according to
the criteria of viability criteria. This system also provides a graphic representation of
machined part, the forged part and the finished stamp.

Keywords Hot forging. Forged components. Design for manufacturing. Viability. Optimization.

(•) Trabajo recibido el día 31 de marzo de 2000 y aceptado en su forma final el día 29 de septiembre de 2000.

${ }^{*}$ E.U. Ingeniería Técnica Industrial, Universidad Politécnica de Madrid. C/ Ronda de Valencia, 3. 28012 Madrid (España).

${ }^{* *}$ E.T.S.Ingenieros Industriales, UNED. C/ Ciudad Universitaria s/n. 28040 Madrid (España).

$\left(^{* * *}\right)$ E.T.S. Ingenieros Industriales, Universidad de Castilla-La Mancha. C/ Campus Universitario s/n. 13071 Ciudad Real (España). 


\section{INTRODUCCIÓN}

El diseño de piezas industriales supone un claro ejemplo de esfuerzo integrador que, con un enfoque interdisciplinar, requiere un análisis detallado de los diferentes conocimientos científicos y tecnológicos implicados en su concepción y desarro1lo. En esta línea, se sitúan los esfuerzos del denominado "Diseño para la Fabricación y Montaje"[1], cuyo objetivo es crear, ya durante la fase de diseño, un producto fácil de fabricar y montar, reduciendo con ello los costes asociados.

El análisis de todos los factores anteriormente expuestos puede resultar extremadamente complejo, dificultando la obtención de soluciones óptimas. Por ello, se han desarrollado diferentes enfoques para facilitar la toma de decisiones que, con un trasfondo fundamentalmente económico, se centran en el ciclo de vida de los materiales. Estas orientaciones alcanzan desde los niveles estratégicos y tácticos de la empresa (políticas de inversión, nuevas tecnologías o materiales, flexibilidad, etc.) hasta los propiamente operativos ${ }^{[2]}$.

Actualmente se están desarrollando orientaciones de análisis que, bajo la denominación de "Módulos Técnico-Económicos" (MTE), abordan las variaciones de los modelos de procesos convencionales con especial atención a la evaluación de las implicaciones de costes, variables de procesos y parámetros económicos. Es decir, los MTE proporcionan un método que considera el material como una entidad cuyo valor económico varía según el flujo de trabajo, siendo los factores que afectan a cada fase del proceso de fabricación una función del diseño del producto ${ }^{[3]}$.

En este sentido, el presente trabajo presenta diferentes criterios de viabilidad y adecuación que permiten reducir el periodo de análisis de las distintas alternativas de diseño de la pieza estampada, optimizando la decisión final. Además, posibilita la disminución de los tiempos de respuesta en la comunicación interactiva de los diferentes factores que intervienen en la estampación, facilitando su coordinación.

\section{EL PROYECTO DE PIEZAS ESTAMPADAS}

En el conformado por deformación las características de las estampas o matrices empleadas determinan, unívocamente, tanto la forma final como la precisión de las piezas obtenidas ${ }^{[4]}$.

Una de las principales consideraciones analizadas en el diseño de una pieza estampada es el costo total de fabricación de la pieza final. Por regla general, se puede descomponer este costo en cuatro grupos: costes de material, de utillaje, de producción y de mecanizado ${ }^{[5]}$. El tratamiento que se proporcione a cada uno de estos factores generará diferentes soluciones alternativas.

Así pues, la obtención de piezas acabadas que proporcionen un servicio óptimo al menor coste posible requiere un estudio integrado que abarque los diferentes elementos implicados en el proyecto de estampación ${ }^{[6]}$.

Con este objeto, se considera conveniente que el cliente presente el plano de la pieza acabada al estampador, a fin de que éste establezca el plano de la pieza estampada. El proyecto de la pieza forjada responde a una serie limitada de reglas o leyes que permiten la existencia de diferentes variantes de ejecución, aunque cada una con un costo determinado. A continuación y, tomando como referencia el plano de.la pieza estampada, se realiza el diseño de la matriz final de acabado. Se elige entre las posibles formas de obtención aquélla que, cumpliendo satisfactoriamente con las exigencias solicitadas a la pieza, resulte más ventajosa.

Con el proceso de deformación en caliente no sólo se pretende proporcionarle la geometría deseada a la pieza sino dotarla de las características, tanto mecánicas como microestructurales, adecuadas a las solicitaciones previstas. Para ello, es preciso seleccionar los parámetros de control del proceso de deformación más apropiados. Estos parámetros pueden ser variables físicas externas (temperatura, velocidad de deformación, etc.) o factores microestructurales (densidad de defectos y su movilidad, formación de subestructuras, tamaño de grano, etc.).

La propiedad más importante de un material durante el proceso de conformado es su conformabilidad, es decir, su capacidad para deformarse plásticamente sin romperse. Esta puede ser intrínseca (función del propio material) o dependiente del estado de tensión (según las características de la tensión aplicada y la forma geométrica de la zona deformada). Ambos aspectos de la conformabilidad deben analizarse con objeto de alcanzar las mejores soluciones y lograr un adecuado proyecto de estampación.

Diferentes estudios ${ }^{[7-8]}$ han abordado la problemática de optimizar la conformabilidad intrínseca mediante la modelización dinámica del material y el uso de mapas de disipación de energía que representan el comportamiento del material cuando está sometido a ciertas condiciones de conformación. 
Bien se trate de aceros o de metales no férreos, el tratamiento térmico es un factor fundamental para garantizar la obtención de las propiedades deseadas en las piezas estampadas. Con ello se consigue la uniformidad del grano o la modificación de la microestructura para que las características mecánicas y físicas queden aseguradas.

En los últimos años, los aceros microaleados han venido aumentando su utilización en diferentes sectores industriales sustituyendo en muchas aplicaciones al acero normal. Ello es debido a que la presencia de elementos microaleantes elimina la necesidad de realizar tratamientos térmicos, lo cual proporciona una reducción de costes. Además, la mejor maquinabilidad de estos aceros origina un aumento de la vida útil de las herramientas y de la eficacia productiva ${ }^{[3]}$.

Las características de resistencia ofrecidas por los aceros microaleados con un contenido medio de carbono son suficientes en la mayoría de las aplicaciones. Sin embargo, la tenacidad presenta, en muchos casos, valores claramente insuficientes. Mejorar esta cualidad requiere conseguir un tamaño fino del grano austenítico. Para ello se emplean dos procedimientos: mantener fino el grano durante la etapa de recalentamiento o modificarlo de forma controlada durante el proceso de conforma$\mathrm{do}^{[9-10]}$.

Una vez que la oficina técnica ha realizado los cálculos y estudios cuantitativos de las solicitaciones a los que estará sometida la pieza y antes de fijar la forma definitiva debe establecerse el número de piezas a fabricar.

La figura 1 ilustra las diferentes formas de estampación adoptadas en función del número de piezas del pedido y el reparto en \% de los factores de coste correspondientes a material, producción, utillaje y mecanizado en un piñón cónico ${ }^{[5]}$.

\section{CRITERIOS DE VIABILIDAD Y OPTIMIZACIÓN}

El estudio de la adecuación de la pieza al proceso de estampación es, en general, un aspecto poco tratado en la bibliografía clásica. Destacan, por su interés en este campo, las aportaciones de Al$\tan ^{[11]}$.

Con el fin de valorar cuantitativamente el grado de adecuación al proceso de forja, Altan sugiere el uso del llamado "factor de dificultad de forma" ("shape difficulty factor") que aplicable a piezas axisimétricas, se define del siguiente modo:

$$
S=K \cdot \beta
$$

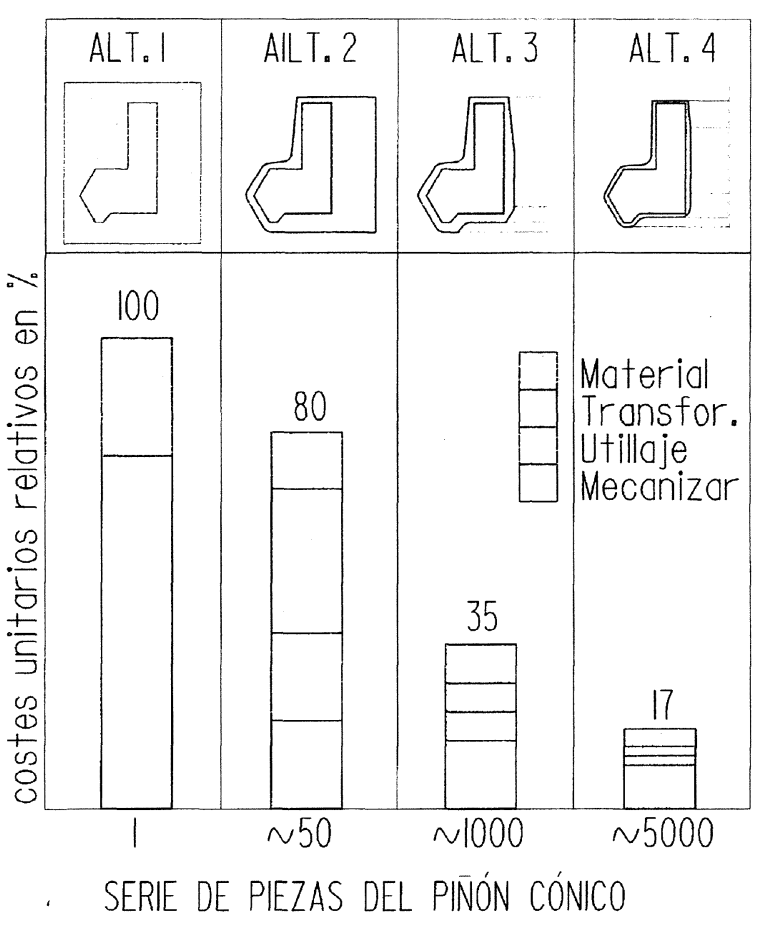

Figura 1. Costes en función de la forma de estampación y la serie.

Figure 1. Costs in function in the wrought shape and the series.

donde

$$
\mathrm{K}=\mathrm{X}_{\mathrm{f}} / \mathrm{X}_{\mathrm{c}}
$$

con

$$
X_{f}=\frac{P^{2}}{F}
$$

y

$$
X_{c}=\frac{P_{c}^{2}}{F_{c}}
$$

y

$$
\beta=2 R_{\mathrm{g}} / R_{\mathrm{c}}
$$

siendo:

$P=$ perímetro de la sección longitudinal axial.

$F=$ superficie de la sección longitudinal axial.

$P_{c}=$ perímetro de la sección longitudinal axial del cilindro envolvente de la pieza.

$F_{c}=$ Superficie de la sección longitudinal axial del cilindro envolvente.

$R_{g}=$ Distancia radial entre el eje de simetría y el centro de gravedad de la media sección de la pieza.

$R_{c}=$ Radio del cilindro envolvente. 
Este factor expresa la complejidad de la estampación de la pieza analizada respecto a la del cilindro envolvente.

Lange ${ }^{[12]}$ también aborda esta temática para exponer una ordenación morfológica según la dificultad de estampación y una propuesta de clasificación de las piezas obtenidas en máquinas de forja horizontal basada en los siguientes criterios: forma básica, dirección de accionamiento, simetría, número de zonas de estampación y dimensión de cuerpos cilíndricos alargados.

Sin embargo, se hace necesario abordar la problemática del análisis y evaluación del grado de adecuación al proceso de estampación desde una perspectiva integradora que tenga en cuenta todos los factores técnico-económicos implicados en el proyecto.

En primer lugar, este análisis debe permitir evaluar la morfología de la pieza estampada, eliminando aquellas geometrías que, por sus implicaciones técnico-económicas, carezcan de viabilidad o interés desde el punto de vista de la tecnología del proceso de conformación.

El análisis efectuado ha puesto de manifiesto ciertas relaciones preferentes entre cualidades o dimensiones geométricas y condicionantes técnicoeconómicos fundamentales para el proceso de estampación en caliente, permitiendo proponer los siguientes criterios limitativos:

a) Acotación del esfuerzo de estampación.

El criterio propuesto se fundamenta en consideraciones energéticas relacionadas con la deformación plástica que aseguran que el esfuerzo deformativo máximo mantenga valores acotados. En el caso de preformas de partida cilíndricas este criterio puede formularse del siguiente modo $^{[13]}$ : "en procesos de estampación, el esfuerzo de deformación mantiene valores minimizados cuando el diámetro de la pieza es igual o menor que el doble de la altura de la misma". Es decir:

$$
2 h \mathrm{i} \geq \mathrm{di}
$$

donde di y hi corresponden con el diámetro y altura de la barra cilíndrica utilizada como material de partida.

b) Esbeltez del material de partida.

Limitando la altura se evita la aparición de pliegues en la distribución de las fibras por efecto del pandeo lateral.
Con este fin resulta aconsejable que en el caso de utilizar barra cilíndrica como material de partida la altura sea inferior al triple del diámetro $^{[14]}$. Es decir:

$$
h \mathrm{i} \leq 3 \cdot d \mathrm{i}
$$

donde di y hi corresponden con el diámetro y altura del cilindro de partida.

c) Dificultad de ejecución específica.

Existen diseños que globalmente presentan geometrías cuya obtención por estampación no es posible. Por ejemplo, cuando existen contrasalidas que se oponen al movimiento de las matrices, de tal forma que al proceder a su apertura, la rotura de la pieza resulta inevitable.

Pero, además es necesario evaluar zonas concretas de la morfología de la pieza estampada para analizar su mayor o menor dificultad de realización. Por ello este criterio limitativo, basado en lo estipulado en la Norma UNE $36203^{[15]}$, considera que la estampación de piezas que incluyen discos de poco espesor, con altura/diámetro $<0,2 \mathrm{o}$, cilindros alargados, cuya altura/diámetro $>2$, presentan una dificultad de ejecución superior al resto de la pieza. Por tanto, requieren un especial tratamiento y atención, lo que aconseja su modificación o la utilización de otro procedimiento de fabricación.

El costo total de la pieza final obtenida por estampación está integrado por los siguientes conceptos: material, transformación, utillaje y mecanizado. Por tanto, el análisis efectuado ha seleccionado, entre los parámetros geométricos característicos de pieza estampada y acabada, áquellos que tienen una relación directa con los costes asociados al proceso de conformación, permitiendo proponer los siguientes criterios de optimización:

a) Dificultad de forma.

Uno de los objetivos característicos del proyecto de estampación consiste en obtener la forma final deseada al menor coste.

La norma UNE $36203^{[15]}$ establece el denominado "coeficiente de dificultad de forma" (CDF), definido como "la relación entre el peso de la pieza estampada y el del sólido envolvente". Dicha relación también puede expresarse en función de los volúmenes correspondientes del siguiente modo:

$$
\mathrm{CDF}=\mathrm{Ve} / \mathrm{Vs} \mathrm{n}
$$


siendo Ve el volumen de la pieza estampada y Vs el volumen del sólido geométrico imaginario que envuelve a la pieza.

b) Aprovechamiento de material.

El grado de semejanza entre la geometría de la pieza estampada y la final puede considerarse como un referente de la cantidad de material que hay que eliminar durante el maquinado $y$, por tanto, puede tomarse como un índice representativo de los costes asociados con el mecanizado de la pieza.

Con este fin, se propone el denominado "coeficiente de aprovechamiento de material" que, representado por CAM, se define como la relación entre los volúmenes de la pieza final y estampada ${ }^{[16]}$. Es decir:

$$
\mathrm{CAM}=\mathrm{Vm} / \mathrm{Ve}
$$

siendo $V \mathrm{~m}$ el volumen de la pieza mecanizada y Ve el volumen de la pieza estampada.

c) Costes de material.

Según se ha comentado anteriormente, en los estudios previos del proyecto se debe poner de manifiesto cuál es el material más adecuado en función de la conformabilidad y las solicitaciones a las que estará sometida la pieza.

En general, puede considerarse que el costo de material asociado a la obtención de una pieza por estampación es directamente proporcional al peso o volumen del tocho de partida utilizado En esta línea se propone el denominado coeficiente del coste de material (CCM). Su expresión es la siguiente:

$$
\mathrm{CCM}=\mathrm{Vsim} / \mathrm{Vsi}
$$

donde

Vsim: menor volumen del sólido inicial entre todas las alternativas

Vsi: volumen del sólido inicial de la alternativa considerada.

Con carácter complementario al índice anterior y para aquellas situaciones donde el material requerido sea aproximadamente el mismo, resulta conveniente plantear un criterio que permita evaluar el aprovechamiento que se hace del material durante el proceso de estampación y mecanizado. Para ello, se propone el coeficiente de rendimiento de material (CRM) definido del siguiente modo:

$$
\mathrm{CRM}=\mathrm{Vm} / \mathrm{Vsi}
$$

donde

Vm: volumen de la pieza mecanizada

Vsi: volumen del sólido inicial de partida

c) Orientación del fibrado.

Los lingotes de material se laminan por diferentes pasadas sucesivas para producir barras y/o tochos con una orientación fibrosa que se extiende a lo largo de la barra de la que se extraerá el material de partida para la forja.

Esta característica asegura un buen comportamiento técnico del material cuando está sometido a solicitaciones en sentido longitudinal. Puesto que las operaciones de forja no modifican sustancialmente esta orientación, las piezas estampadas presentan también esta cualidad. Por ejemplo, en la realización de piezas de revolución, las fibras del tocho de partida se orientan en sentido axial, consiguiendo, en la estampación, una simetría perfecta del fibrado en relación a su eje y unas mismas características técnicas sobre puntos homólogos en todas las generatrices de la piezas.

Por tanto, al proyectar una determinada pieza estampada resulta fundamental hacerlo de manera que las fibras del material se distribuyan por la pieza paralelamente a las direcciones de los esfuerzos de tracción que debe soportar la pieza en servicio $^{[18]}$. De esta forma, para una misma sección transversal, las piezas estampadas presentan mejor resistencia a la tracción que las obtenidas por otros procesos tecnológicos. En la norma UNE $36202^{[19]}$, relativa a las condiciones técnicas generales de suministro de piezas estampadas en caliente, se reconoce la trascendencia de la orientación de las fibras al fijar las características que debe presentar el fibrado y recoger el sistema para especificar su orientación.

En el caso de piezas con simetría de revolución, este criterio puede cuantificarse en el denominado coeficiente de aprovechamiento del fibrado (CAF) definido del siguiente modo:

$$
\mathrm{CAF}=1-\frac{2|\alpha-\beta|}{\pi}
$$

donde

K: ángulo predominante de orientación del fibrado obtenido en la estampación.

2: ángulo de orientación del fibrado deseable en función de las solicitaciones.

Así pues, los criterios expuestos facilitan tomar decisiones en la fase de proyecto sobre las 
morfologías que deben ser excluidas del proceso de conformación y además, los coeficientes CDF, CAM, CCM, CRM y CAF permiten evaluar cuantitativamente el grado de adecuación al proceso, de diferentes alternativas analizadas.

\section{APLICACIONES}

Con objeto de ilustrar la aplicación de los criterios de viabilidad y adecuación propuestos y mostrar las ventajas de su utilización en la selección de la mejor alternativa se ha desarrollado una aplicación comparativa en la tipología correspondiente a las piezas cilíndricas axisimétricas de varios cuerpos. En la figura 2 se muestra una pieza del tipo considerado y su correspondiente pieza forjada, diseñada
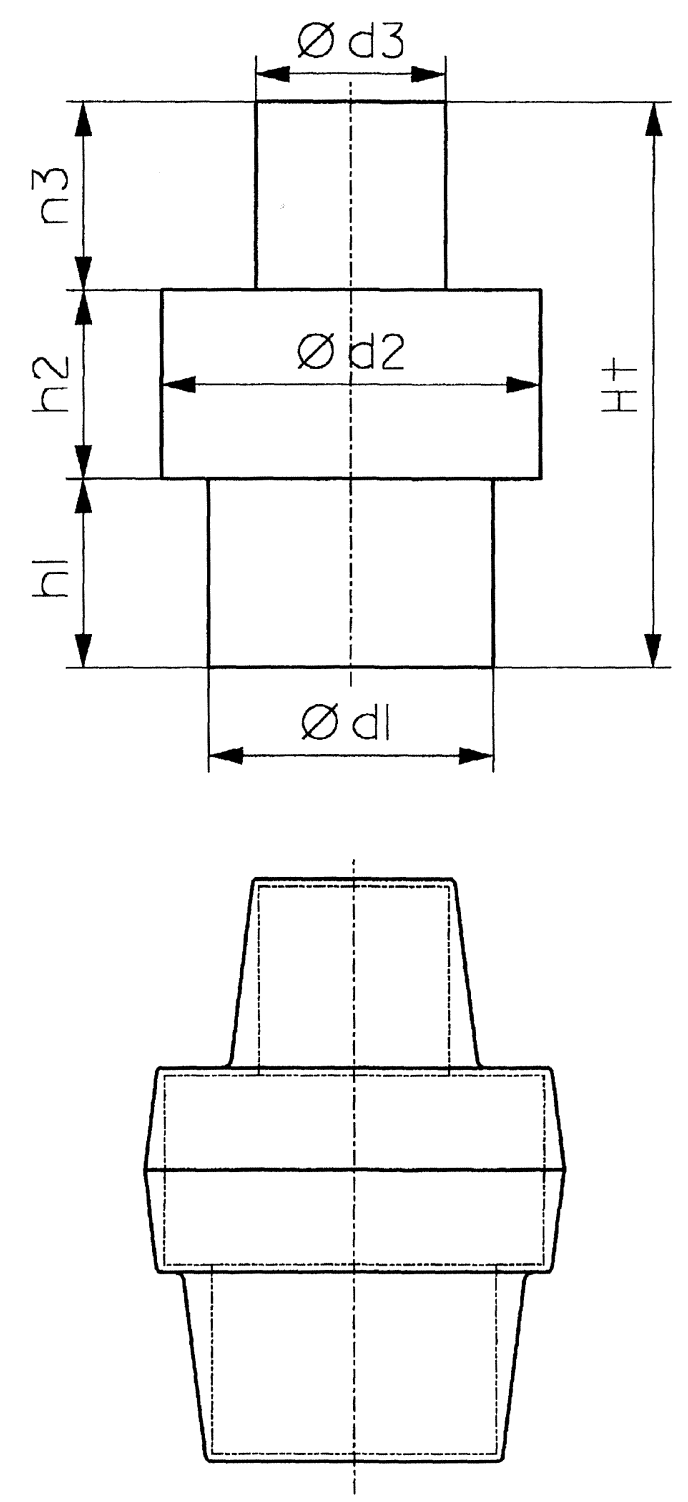

Figura 2. Pieza final y estampada.

Figure 2. Finished and forged part teniendo en cuenta las normas que rigen los procesos de estampación en caliente.

En la aplicación desarrollada se incluyen como posibles materiales el aluminio y el acero. En este último caso, con el fin de establecer las tolerancias dimensionales y delimitar el esfuerzo deformativo, se distingue entre aceros muy aleados $(\mathrm{C} \geq 0,65 \%$ y elementos de aleación $\geq 5 \%$ ) y poco aleados ( $C<0,65 \%$ y elementos de aleación $<5 \%$ ).

La selección de las relaciones más interesantes, desde el punto de vista de optimización del proceso de estampación, precisa fijar el sentido del esfuerzo de deformación. Así, en el caso de piezas de revolución resulta más conveniente realizar el estampado con el eje del material en posición vertical, puesto que el mecanizado de las matrices se efectúa con mayor facilidad y economía. Por tanto, al establecer una dirección para el esfuerzo coaxial al eje de la pieza, la superficie de separación será un plano horizontal perpendicular al sentido de dicha acción. Además, este plano debe incluir la sección de la pieza con mayor área. Así pues, para el caso de piezas axisimétricas de varios cuerpos, con esfuerzo deformativo coaxial al eje del material, la superficie de separación es un plano horizontal localizado en el cuerpo de mayor diámetro ${ }^{[18-20]}$.

Teniendo en cuenta la información anterior y considerando los datos de la pieza final mecanizada se determinan las características geométricas de la pieza estampada, según los códigos de diseño de la bibliografía existente al efecto ${ }^{[21]}$.

Con objeto de acortar períodos de análisis y respuesta se ha desarrollado un sistema informático que simula geométricamente el proceso de estampación, permitiendo calcular los coeficientes de adecuación en aquellas tipologías que sean posibles según los criterios de viabilidad. Este sistema también proporciona una representación gráfica preliminar de pieza mecanizada, pieza estampada y matriz de acabado. Para ello se ha realizado un interfaz operativo en base a primitivas geométricas que asegura su comunicación con un sistema de $\mathrm{CAD}^{[22]}$.

La aplicación desarrollada se ha centrado en el análisis del diseño de una pieza con simetría de revolución de tres cuerpos. Los estudios previos correspondientes han puesto de manifiesto, al estudiar las solicitaciones a las que estará sometida la pieza, que la utilización del proceso de estampación resulta una alternativa interesante puesto que permite aprovechar todas las ventajas inherentes a la orientación del fibrado $(\mathrm{CAF}=1)$. Sin embargo, al tomar en consideración su articulacíón y montaje con otras piezas del sistema 
mecánico, se plantean varias alternativas dimensionales viables. Así, se obtiene la siguiente configuración (dimensiones en $\mathrm{mm}$ ):

$$
d_{1}=70 ; \quad d_{2}=100 ; \quad d_{3}=50 ; \quad h_{2}=50 ; H_{\mathrm{t}}=150 \text {. }
$$

En esta aplicación han quedado sin determinar las alturas $h_{1}$ y $h_{3}$ y será necesario fijar cuáles deben ser sus valores para, sin variar la altura total (150 $\mathrm{mm}$ ), optimizar la adecuación de la pieza al proceso de estampación.

En las figuras 3 y 4 se muestran los resultados obtenidos al introducir los parámetros anteriores en el sistema informático. En dichos gráficos se muestran las curvas de los coeficientes CDF, CAM, CCM y CRM en función de las dimensiones variables. Por otra parte, y con objeto de evaluar globalmente los tres coeficientes de adecuación (CDF, CAM y CCM) se propone la utilización del siguiente índice (coeficiente de adecuación global):

$$
\mathrm{CAG}=k_{a m} \mathrm{CAM}+k_{d f} \mathrm{CDF}+k_{c m} \mathrm{CCM}
$$

donde los coeficientes $k_{a m}, k_{d f}$ y $k_{c m}$ representan el peso relativo de cada criterio de adecuación en el costo total de la pieza final. Para el caso analizado, los valores aproximados de dichos coeficientes son los siguientes ${ }^{[5]}$ :

$$
k_{a m}=0,5 \quad k_{d f}=0,3 \quad k_{c m}=0,2
$$

En la figura 5 se ha representado la curva del CAG para la aplicación considerada. De su

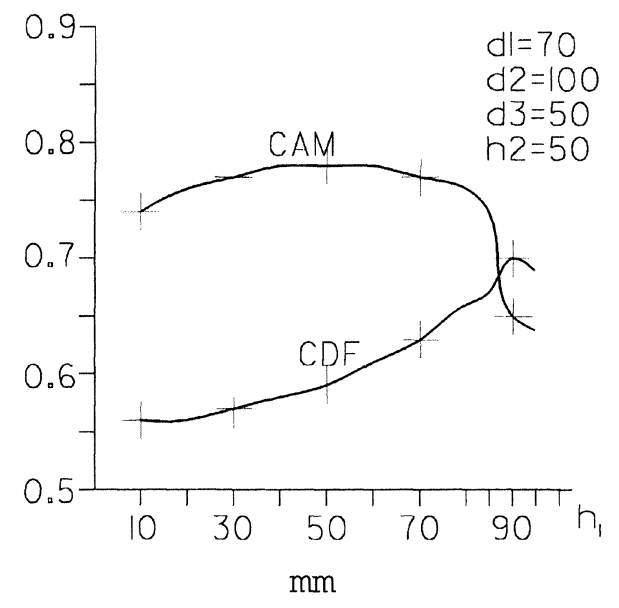

Figura 3. Curvas de los coeficientes CDF y CAM para $h 2=$ $50 \mathrm{~mm}$.

Figure 3. Curves of the coefficients $C D F$ and $C A M$ for $h 2=$ $50 \mathrm{~mm}$.

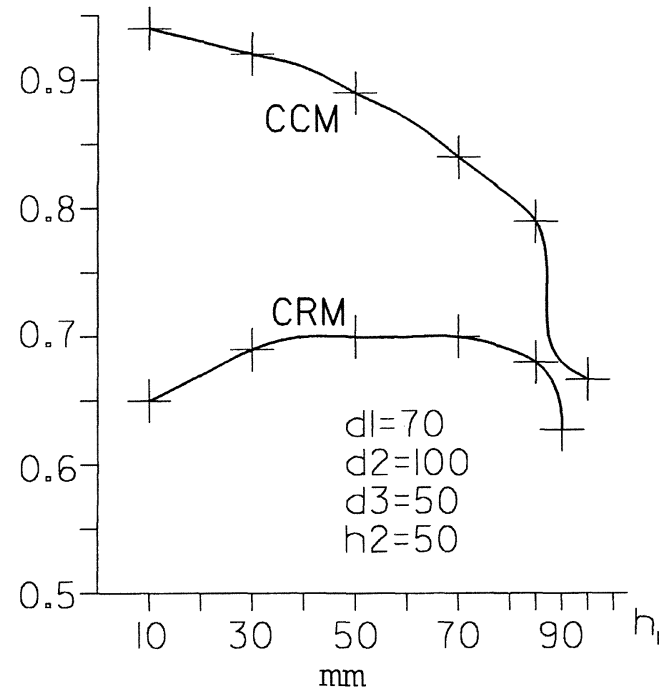

Figura 4. Curvas de los coeficientes $C C M$ y $C R M$ para $h 2=$ $50 \mathrm{~mm}$.

Figure 4. Curves of the coefficients CCM and CRM for $h 2=$ $50 \mathrm{~mm}$.

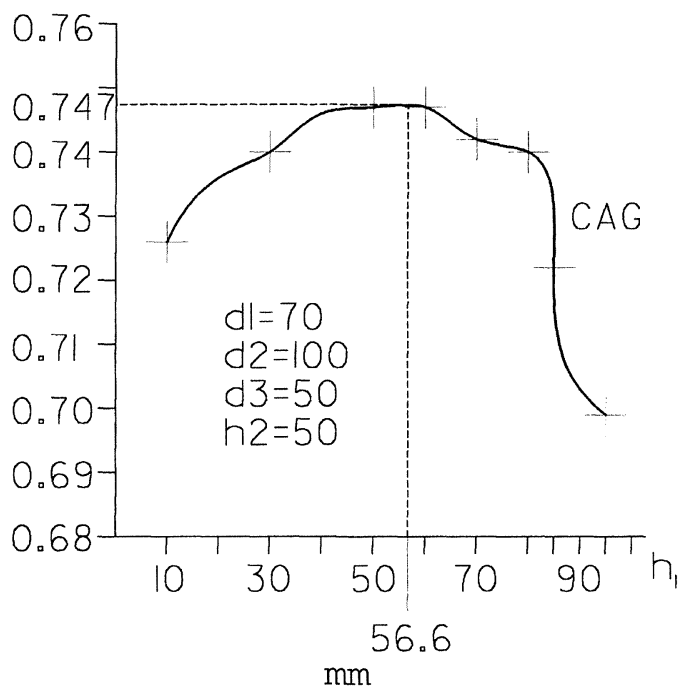

Figura 5. Curvas del coeficiente $C A G$ para $\mathrm{h} 2=50 \mathrm{~mm}$.

Figure 5. Curve of the coefficient $C A G$ for $h 2=50 \mathrm{~mm}$.

análisis se deduce que presenta un máximo en $h_{1}=56.6 \mathrm{~mm}$, tomando CAG el valor 0,747. Para esta altura del cuerpo inferior los valores de los criterios de adecuación son los siguientes:

$$
\begin{gathered}
\mathrm{CAM}=0,78 ; \mathrm{CDF}=0,60 ; \mathrm{CCM}=0,88 \\
\mathrm{y} \mathrm{CRM}=0,70
\end{gathered}
$$

Por ello, la elección de las dimensiones $h 1=56.6 \mathrm{~mm}$ y $h_{3}=43,4 \mathrm{~mm}$ permite, además de cumplir las especificaciones establecidas en el diseño de la pieza, optimizar su fabricación por estampación. 
Las figuras 6,7 y 8 proporcionadas por el sistema informático muestran las representaciones gráficas correspondientes a pieza final, matriz de acabado y pieza estampada de la solución elegida, respectivamente.

\section{CONCLUSIONES}

El presente trabajo sienta las bases para el desarrollo de un método eficaz para el diseño de piezas estampadas en caliente, que tenga en cuenta las características del proceso de conformación, permitiendo optimizar la decisión final.

Con este fin, se han establecido pautas de actuación para discernir la viabilidad y adecuación de las piezas consideradas al proceso de forja. Así, se propone la realización previa de un análisis de la pieza mecanizada que evidencie sus características geométricas y las formas de estampación correspondientes. El análisis técnico-económico de dichas morfologías permite delimitar aquellas situaciones inadecuadas para obtener por estampación. Para ello, se introducen tres criterios de carácter excluyente: acotación del esfuerzo de estampación, esbeltez del material de partida y dificultad de ejecución específica. Pero además, los coeficientes de optimización propuestos (CAM, CDF, CCM y CRM) posibilitan cuantificar, en aquellas tipologías viables, el grado de adecuación al proceso de forja, facilitando la toma de decisión.

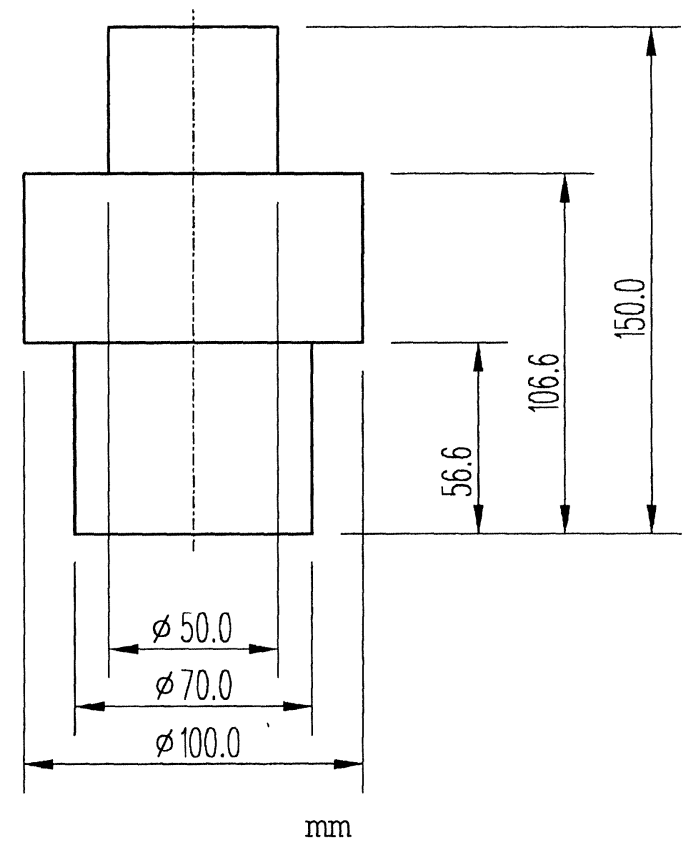

Figura 6. Pieza mecanizada para la solución $\mathrm{h} 1=56.6 \mathrm{~mm}$.

Figure 6. Finished part for the solution $h 1=56.6 \mathrm{~mm}$.

Rev. Metal. Madrid 36 (2000)

(c) Consejo Superior de Investigaciones Científicas

Licencia Creative Commons 3.0 España (by-nc)

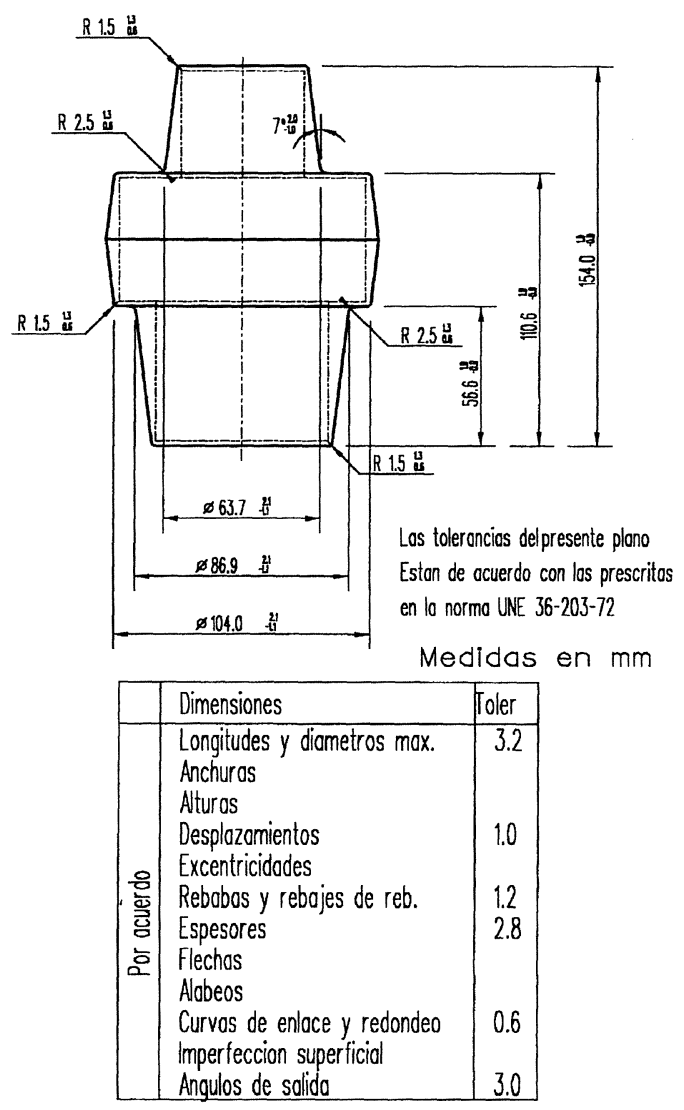

Figura 7. Pieza estampada con sus tolerancias dimensionales.

Figure 7. Forged part with their dimensional tolerances.

Fijadas estas bases y con objeto de acortar tiem-

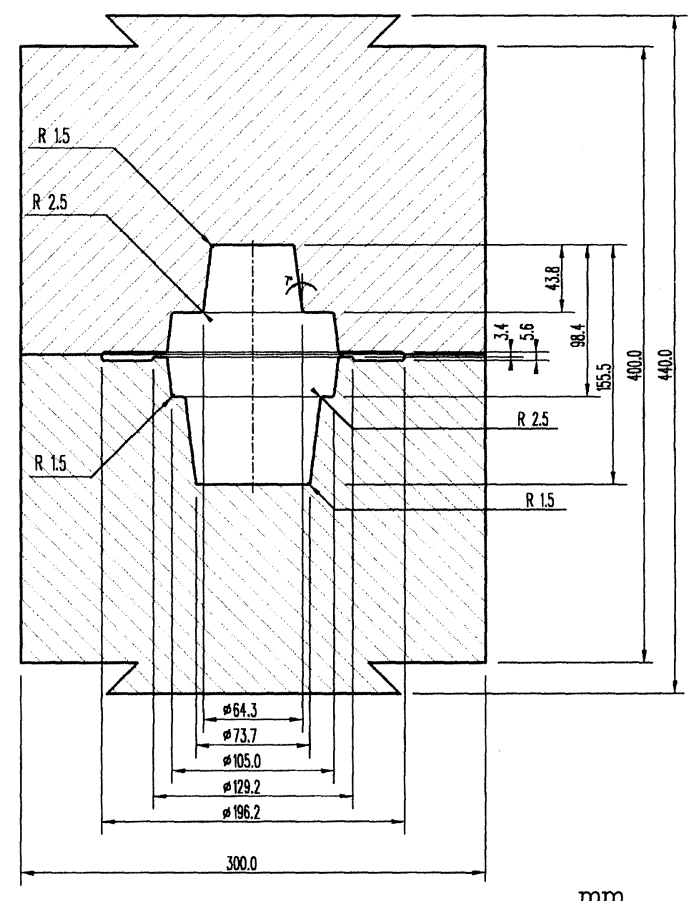

Figura 8. Estampa de acabado.

Figure 8. Finishing Stamp. 
Fijadas estas bases y con objeto de acortar tiempos de respuesta y análisis en una aplicación correspondiente a piezas axisimétricas, se ha introducido un sistema informatizado que, tomando como dato de partida el plano de mecanizado de la pieza, simula geométricamente el proceso de estampación, permitiendo calcular los coeficientes de adecuación en aquellas tipologías que son posibles según los criterios de viabilidad. Este sistema también proporciona una representación gráfica de pieza mecanizada, pieza estampada y matriz de acabado.

\section{REFERENCIAS}

[1] G. Bootthoyd, P. Dewhurst y W. Knight, Product design for manufacture and assembly, Ed. Marcel Deckker, Nueva York, EE.UU., 1994, pp.1-27.

[2] J.A. Domínguez, M.J. Alvarez, M.A. Domínguez, S. García y A. Ruiz, Dirección de operaciones, Ed. McGraw-Hill, Madrid, España, 1995, pp.141-172.

[3] F. Faura, Rev. Metal. Madrid 32 (1996), 381-390.

[4] F. Fadón, M. Domínguez, M.A. Sebastián, III Congreso de Expresión Gráfica en la Ingeniería, vol. 1, Las Palmas de Gran Canaria, España, 1991, Comité Local de III Congreso, U. de Las Palmas, Las Palmas de Gran Canaria, España, 1991, pp. 1-9.

[5] Euroforge, Proyecto y diseño de las piezas forjadas por estampación, Ed. Sife, Bilbao, España, 1992, pp.1-27.

[6] J.M. Arenas, F. Faura y M.A. Sebastián, Deformación Metálica 215 (1994) 20-28.

[7] J.M. Cabrera y J.M. Prado, Rev. Metal. Madrid 33 (1997) 80-88.

[8] J.M. Cabrera y J.M. Prado, Rev. Metal. Madrid 33 (1997) 153-160.

[9] A. Alomar y J.M. Prado, Rev. Metal. Madrid 33 (1997) 89-100.

[10] J.M. Cabrera, A. Alomar y J.M. Prado, Rev. Metal. Madrid 33 (1997) 143-152.
[11] T. Altan, S. OH. y M. Gegel, Metal forming: fundamentals and applications, American Society for Metals, Metal Park, EE.UU, 1983, pp. 145-151.

[12] K. LANGE, Handbook of metal forming, Ed. McGraw-Hill, Nueva York, EEUU, 1985, pp. 11.1-11.85.

[13] M. Domínguez, J.M. Arenas, F. Fadón y M.A. Sebastián, IV Congreso de Expresión Gráfica "Ingeniería Gráfica”, vol.1, Madrid, España, 1992, Ed. Ingegraf, U. Politécnica de Madrid, Madrid, España, 1992, pp. 26. 1-26.13.

[14] J. Billigmann y M.D. Feldmann, Estampado y prensado a máquina, Ed. Reverté, Barcelona, España, 1979, pp. 513-528

[15] AENOR, UNE 36203-72: Piezas de acero estampadas en caliente (tolerancias dimensionales), AENOR, Madrid, España, 1972, pp. 2-17.

[16] J.M. Arenas, C. González y M.A. Sebastián, XI Congreso Nacional de Ingeniería Mecánica, vol.2, Valencia, España, 1994, Ed. Asociación Española de Ingeniería Mecánica, U. Politécnica de Valencia, Valencia, España, '1994, pp. 213-219.

[17] A. Chamouard y J. Foucher, Conception e tracé des pièces estampèes, Ed. ADETIEF, París, Francia, 1979, pp. 1-21.

[18] AENOR, UNE 36 202-78: Piezas de acero estampadas en caliente (condiciones técnicas generales de suministro), AENOR, Madrid, 1978, pp. 2-7.

[19] R. Diefrich, G. Facy, E. Hugonnaud, M. Pompidou y J.P. Trotignon, Précis de construction mécanique, Ed. AFNOR, 4ª edición, París, Francia, 1979, pp. 48-63.

[20] M. Rossi, Estampado en caliente de metales, Ed. Hoeppli/Científico-Médica, Barcelona, España, 1971, pp.110-135.

[21] J.M. Arenas, F. Fadón y M. Sebastián, V Congreso Internacional de Expresión Gráfica "Diseño Industrial", vol.1, Gijón, España, 1993, Ed. Ingegraf, U. de Oviedo, Gijón, España, 1993, pp. 117-126.

[22] M. Domínguez, J. Conde, J.L. Borrego, M.M. Espinosa, F. Fadón, J. Pose, J.M. OchoA, J.M. SAnz, P. Cruz, A. Caño y J.M. Arenas, Diseño y dibujo asistido por ordenador, UNED, Madrid, España, 1995, pp. 229-249. 